\title{
Glückwunsch und Widmung
}

\section{Michael Ermann}

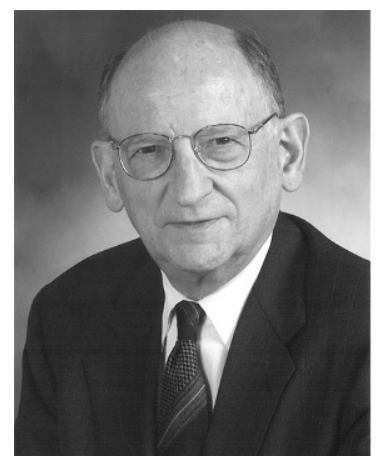

Otto F. Kernberg

\section{Lieber Otto F. Kernberg!}

Wir, die Herausgeber des Forums der Psychoanalyse, widmen Ihnen dieses Heft zu Ihrem 90. Geburtstag.

Am 12. September blicken Sie auf 90 Jahre Ihres Lebens zurück. Was für ein Leben! Es hat Sie durch alle Kontinente an Brennpunkte dieser Erde und in verborgene Winkel geführt. Sie mussten die Tiefen des Schicksals durchschreiten, aber Sie konnten auch den Glanz des Erfolges erfahren und ihn auskosten.

Prof. Dr. M. Ermann $(\bowtie)$

Innsbrucker Str. 56, 10825 Berlin, Deutschland

E-Mail: michael.ermann@t-online.de 
Aus der Sicht unseres Faches, der Psychoanalyse, haben Sie wertvolle Schätze an Erkenntnissen, Erfahrungen und Wissen angehäuft und mit Ihrem unermesslichen Fleiß und Ihrer Begabung als Lehrer an andere weitergegeben. Damit haben Sie Generationen von Analytikerinnen und Analytikern in vielen Ländern bereichert.

Nicht zuletzt bei uns in Deutschland waren Sie seit Jahrzehnten ein unermüdlicher Kämpfer für die Erneuerung der Psychoanalyse. So konnten wir Sie auch bewegen, ohne Zögern unsere Einladung anzunehmen, diese Zeitschrift als Beirat von Anbeginn zu unterstützen. Dafür und für die Beiträge, die Sie im Forum der Psychoanalyse publiziert haben, danken wir Ihnen.

Wir wünschen Ihnen viele gesunde, unbeschwerte und sicherlich noch ertragreiche Jahre!

Cord Benecke, Anna Ursula Dreher, Michael Ermann, Jürgen Körner, Ulrich Lamparter, Diana Pflichthofer und Timo Storck 